\title{
Synthesis of a vinylogue tetrathiafulvalene derivative and study of its charge transfer complex with $\mathrm{TCNQF}_{4}$
}

\author{
Tommaso Salzillo ${ }^{\mathrm{ab}}$, Núria Crivillers ${ }^{\mathrm{a}}$, Marta Mas-Torrent ${ }^{\mathrm{a}}$, Klaus Wurst, ${ }^{\mathrm{c}}$ Jaume \\ Veciana ${ }^{a}$, Concepció Rovira ${ }^{a, *}$ \\ ${ }^{a}$ Department of Molecular Nanoscience and Organic Materials, Institut de Ciència de Materials de \\ Barcelona (ICMAB-CSIC) and (CIBER-BBN), Campus de la UAB, 08193 Bellaterra, Spain \\ ${ }^{b}$ Universidad Autónoma de Barcelona, 08193 Bellaterra (Cerdanyola del Vallès), Spain \\ c Institute of General, Inorganic and Theoretical Chemistry, University of Innsbruck, Innrain 80/82, 6020 \\ Innsbruck, Austria \\ e-mail: cun@icmab.es
}

\begin{abstract}
Efficient syntheses of a vinylogue TTF derivative bearing four cyanoethylsulfanyl substituents, 9 is described. Cyclic voltammetry establishes that the novel compound shows good donor properties and a reduced on-site coulombic repulsion. Single crystal $\mathrm{X}$-ray structure determination shows that the molecule has a planar structure of the conjugated moiety with a slipped $\pi-\pi$ stacking organization in the crystal parallel to the crystallographic $a$ axis. EPR, UV-Vis and IR spectroscopies show that $\mathbf{9}$ forms a charge transfer complex with TCNQF4. EPR studies allow for the recognition of the 3:2 stoichiometry ratio between donor and acceptor while IR spectroscopy gave the estimation of the ionicity of the complex, which was found to be 0.31 .
\end{abstract}

Keywords: TTF vinylogue; semiconductors, charge transfer complex; $\pi-\pi$ stacking; EPR

\section{Introduction}

The field of organic conductors has been continuously evolving from the first observation of high conductivity in an organic molecular complex.[1] The breakthrough that represented the metallic behaviour of the stable donor-acceptor complex tetrathiafulvalene-tetracyanoquinodimethane (TTF-TCNQ)[2] boosted the research on molecular charge transfer (CT) complexes and ion radical salts, giving rise to a pleiad of synthetic metals and superconductors[3] with very interesting electrical, optical and magnetic properties that can be modulated either by external stimuli like temperature or pressure or by chemically changing the nature of the donor and acceptor components[4-7]. In addition, some charge transfer complex crystals have shown ferroelectric properties when the CT dipoles present a long range orientation. [8,9] The donor and acceptor molecules play also a very important role as semiconductors in molecular electronics, $[10,11]$ and recently have also been successfully applied as $p$ - or $\mathrm{n}$ - dopants to modulate the properties of electronic materials. [12-14] All the properties of CT complexes depend on the degree of charge transfer, that can be modulated by the 
difference between the HOMO of the donor and the LUMO of the acceptor, and the control of the supramolecular organization. Therefore, the proper election of the component molecules is very important and it is interesting to have a library of molecular components as large as possible. One family of donors that has been extensively used as semiconductors[15] and as components of CT complexes are TTF derivatives.[16-18] An extensive work has been done on the chemical modification of TTF in order to introduce electronic modifications and substituents promoting specific supramolecular organizations giving rise to the desired orbital overlap. [19-21] Among them, the vinylogous of TTF derivatives are less developed even though the incorporation of conjugated alkene linking groups between the two 1,3-dithiole rings of TTF should reduce the intramolecular Coulombic repulsions and promote the spatial extension of the $\pi$-framework. Indeed, the already reported TTF and Tetraselenafulvalene (TSF) vinyloguous derivatives show a low second oxidation potential and extended planar structures.[22-24,24-26]

In this context we report here the synthesis, spectroscopic and structural characterization of a new TTF vinylogue derivative (TTFV 9, Scheme 1). So far our studies have shown that, as projected, the difference between the first and second oxidation potentials, which is only of $0.17 \mathrm{~V}$, should derive in a lowering of the Coulombic repulsion. Further, the X-ray crystal structure reveals a planar $\pi$-extended system that crystallises in one-dimensional TTF stacks. The study of the charge transfer complex with $\mathrm{TCNQF}_{4}$ is also described.

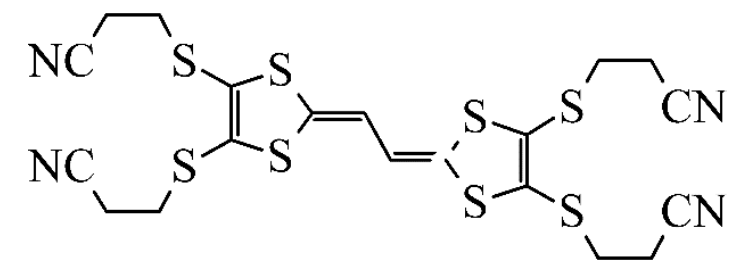

Scheme 1 Molecular structure of the TTF vinylogue derivative 9

\section{Experimental methods}

\subsection{General Details.}

All reactions were carried out using solvents which were dried by routine procedures. Starting compounds were purchased from Sigma Aldrich and used without further purification.

Infrared (IR) spectra were obtained in transmission mode with a Jasco 4700 Fourier transform (FTIR) spectrometer in the spectral range from $400-4000 \mathrm{~cm}^{-1}$ with a resolution of $4 \mathrm{~cm}^{-1}$ and 32 scans. Absorption spectra of the solutions and of the solid samples were obtained from a Varian UV-Vis-NIR Cary 5000 spectrophotometer. For both IR and UV-Vis measurements the powders of the pristine TCNQF 4 and TTFV 9and the crystals of the charge transfer complex were dispersed in a $200 \mathrm{mg}$ of spectroscopic grade $\mathrm{KBr}$ and used as pellet. ${ }^{1} \mathrm{H}$ and ${ }^{13} \mathrm{C}$ NMR spectra were recorded at 250 or $400 \mathrm{MHz}$ in a Bruker Advance apparatus. The following abbreviations for stating the multiplicity 
of the signals have been used: $s$ (singlet), bs (broad singlet), $d$ (doublet), $t$ (triplet), bt (broad triplet), st (pseudotriplet), $m$ (multiplet), and q (quaternary carbon atom). Chemical shifts refer to signals of tetramethylsilane in the case of ${ }^{1} \mathrm{H}$ and ${ }^{13} \mathrm{C} N M R$ spectra. EPR spectra were obtained with a Bruker 300 spectrometer equipped with a TE 102 microwave cavity, a variable temperature unit, and a field frequency lock system, and line positions were determined with a NMR gaussmeter. The modulation amplitude was kept well below the line width, and the microwave power was well below saturation. The cyclic voltammetric measurements were performed on a potentiostat/galvanostat 263a of EG\&G Princeton Applied Research controlled by a personal computer and driven by dedicated software with a conventional threeelectrode configuration consisting of platinum working and auxiliary electrodes and an $\mathrm{Ag} / \mathrm{AgCl}$ reference electrode. The experiments were carried out with a $10^{-3} \mathrm{M}$ solution of the TTFV in dry $\mathrm{CH}_{2} \mathrm{Cl}_{2}$ containing $0.1 \mathrm{M}$ of $\left[(\mathrm{n}-\mathrm{Bu})_{4} \mathrm{~N}\right] \mathrm{PF}_{6}$ as supporting electrolyte. Deoxygenation of the solutions was achieved by bubbling nitrogen for at least $10 \mathrm{~min}$. Time-of-flight secondary ion mass spectrometric (ToF-SIMS) measurements were recorded under the following specific analysis conditions: primary gun energy $25 \mathrm{KV}$, extractor energy $8.5 \mathrm{KV}$, emission current $1.05 \mathrm{~mA}$, employing $\mathrm{Bi}^{3+}$ with an intensity of the primary ions of $0.25 \mathrm{pA}$. The experiments were performed in vacuum at $8.3 \cdot 10^{-9}$ mbar. The spot size was $200 \cdot 200 \mu \mathrm{m}$, with a resolution of 128.128 pixels, collecting in excess of $1 \cdot 10^{12}$ of ionic intensity per spectrum.

\subsection{Synthesis}

Compounds $1,[27] \mathbf{2 , [ 2 8 ] ~ a n d ~ 3 - 5 , [ 2 9 ] ~ w e r e ~ s y n t h e s i z e d ~ f o l l o w i n g ~ t h e ~ l i t e r a t u r e ~}$ procedures.

\section{Compound 6}

To a solution of $5(0.917 \mathrm{~g}, 1.54 \mathrm{mmol})$ and glyoxal $(2.9 \mathrm{~mL}, 62.2 \mathrm{mmol})$ in acetonitrile $(40 \mathrm{~mL})$, triethylamine $(1.15 \mathrm{~mL}, 8.26 \mathrm{mmol})$ was added drop-wise with stirring at room temperature and in an argon atmosphere. After stirring for 21 h., water $(70 \mathrm{~mL})$ was added and the residue was twice extracted with dichloromethane $(70 \mathrm{~mL})$. The extract was dried over $\mathrm{Na}_{2} \mathrm{SO}_{4}$, and concentrated in vacuum. The orange residue was purified by column chromatography ( silica gel, $\mathrm{CH}_{2} \mathrm{Cl}_{2}$ ) to give an oil that by crystallization from ethyl acetate-propanol gave $0.250 \mathrm{~g}(57 \%)$ of compound 6 as orange microcrystals. ${ }^{1} \mathrm{H} \mathrm{NMR}$ $\left(\mathrm{CDCl}_{3}, \delta \mathrm{ppm}\right): 9.43(\mathrm{~s}, 1 \mathrm{H}, \mathrm{CHO}), 6.70(\mathrm{~s}, 1 \mathrm{H}, \mathrm{CH}), 3.17\left(\mathrm{~m}, 4 \mathrm{H},-\mathrm{SCH}_{2}\right), 2,78(\mathrm{~m}, 4 \mathrm{H}$, $\left.\mathrm{CH}_{2} \mathrm{CN}\right)$. IR $\left(\mathrm{KBr}, \mathrm{cm}^{-1}\right)$ 2925, 2250, 1633, 1501, 1453,1413, 1389,1280, 1040, 964, 810, 752, 556, 472. Anal. Calcd for: $\mathrm{C}_{11} \mathrm{H}_{10} \mathrm{~N}_{2} \mathrm{OS}_{4}$ : C, 42.01; H, 3.21, N; 8.91; O, 5.08; S, 40,79. Found: $\mathrm{C}, 42.14 ; \mathrm{H}, 2.99 ; \mathrm{N}, 8.74 ; \mathrm{O}, 5.45 ; \mathrm{S}, 40.68$.

\section{Compound 7}

To a cold (ice bath) solution of compound $4(1.49 \mathrm{~g}, 4.65 \mathrm{mmol})$ in a mixture of anhydrous ethyl ether $(100 \mathrm{ml})$ and acetic anhydride, was added dropwise $5 \mathrm{~mL}$ (103.01 $\mathrm{mmol}$ ) of tetrafluoroboric acid (54\% in ether). The mixture was then stirred for $1 \mathrm{~h}$. The beige precipitate formed was filtered and washed with ether resulting after drying in 
$1.09 \mathrm{~g}(65 \%)$ of compound 7 . The compound should be stored under argon and was used quickly for the next step. ${ }^{1} \mathrm{H} N M R\left(\mathrm{CDCl}_{3}, \delta \mathrm{ppm}\right): 11.0(\mathrm{~s}, 1 \mathrm{H}, \mathrm{CH}+), 3.49(\mathrm{t}, 4 \mathrm{H}, \mathrm{J}=6.75$ $\mathrm{Hz}), 2.89(\mathrm{t}, 4 \mathrm{H}, \mathrm{J}=6.75 \mathrm{~Hz})$. IR $\left(\mathrm{KBr}, \mathrm{cm}^{-1}\right)$ 2961, 2918, 2250, 1421, 1389,1235, 1083$1038,941,739,522$

\section{Compound 9}

First, the phosphorene derivative 8 was generated by treatment of a solution of 7 (1.022 $\mathrm{g}, 2.83 \mathrm{mmol})$ in dry MeCN with tributylphosphine $(0.9 \mathrm{~mL}, 3.59 \mathrm{mmol})$ in dry acetonitrile $(45 \mathrm{~mL})$ under argon at $20^{\circ} \mathrm{C}$ and stirring for $1 \mathrm{~h}$. To the resulting orange solution, a solution of the aldehyde $6(0.237 \mathrm{~g}, 0.75 \mathrm{mmol})$ in dry MeCN was added followed by the immediate addition of freshly distilled triethylamine $(2.2 \mathrm{~mL}, 15.8 \mathrm{mmol})$. The mixture turned dark red with the formation of a precipitate. After stirring overnight, the solid was filtered and thoroughly washed with $\mathrm{MeCN}$ giving pure compound 9 (319 $\mathrm{mg}, 74 \%$ ) as a yellow powder. $\mathrm{Mp}$ : 198-200 ㅇ․ ${ }^{1} \mathrm{H} N M R\left(\mathrm{CD}_{2} \mathrm{Cl}_{2}, \delta \mathrm{ppm}\right): 5.86(\mathrm{~s}, 2 \mathrm{H},-\underline{\mathrm{H}}=\mathrm{CH}-)$, 3.09 (t, J= $6 \mathrm{~Hz}, 8 \mathrm{H},-\mathrm{SCH} 2), 2,77\left(\mathrm{t}, \mathrm{J}=7 \mathrm{~Hz}, 8 \mathrm{H},-\mathrm{CH}_{2} \mathrm{CN}\right)$. IR $\left(\mathrm{KBr}, \mathrm{cm}^{-1}\right)$ 2922, 2854, 2253, 1526, 1486,1413, 1319,1276, 1235, 955, 894, 809, 782, 728, 590. Anal. Calcd for: $\mathrm{C}_{20} \mathrm{H}_{18} \mathrm{~N}_{4} \mathrm{~S}_{8}: \mathrm{C}, 42.08 ; \mathrm{H}, 3.18, \mathrm{~N} ; 9.81 ; \mathrm{S}, 44,93$. Found: C, 42.29; H, 2.85; N, 9.80; S, 45.40.

\subsection{X-Ray diffraction measurements.}

The diffractometer used was a Nonius Kappa CCD, equipped with monochromated Moк $\alpha$ $(\lambda=0.71073 \AA$ ) radiation. The structure was solved by direct methods and refined by the full-matrix least-squares in $\mathrm{F}^{2}$ method with SHELXTL software[30]. Non-hydrogen atoms were refined with anisotropic displacement parameters. Hydrogen atoms bound to carbon atoms were refined with constraints in calculated positions. Crystallographic data for the reported structure have been deposited with the Cambridge Crystallographic Data Centre. Reference number CCDC 1872305

Table 1. Crystal data and structure refinement for crystal of the TTFV.

$\begin{array}{lll}\text { Empirical formula } & \mathrm{C} 20 \mathrm{H} 18 \mathrm{~N} 4 \mathrm{~S} 8 & \\ \text { Formula weight } & 570.86 & \\ \text { Temperature } & 233(2) \mathrm{K} & \\ \text { Wavelength } & 0.71073 \AA \\ \text { Crystal system } & \text { Triclinic } & \alpha=113.768(2)^{\circ} \\ \text { Space group } & \mathrm{P}-1 \text { (no.2) } & \beta=98.300(2)^{\circ} \\ \text { Unit cell dimensions } & \mathrm{a}=5.1844(2) \AA & \gamma=96.630(2)^{\circ} \\ & \mathrm{b}=15.7517(6) \AA & \\ \text { Volume } & \mathrm{C}=17.2326(7) \AA & \\ \text { Z } & 1250.77(8) \AA 3 & \\ \text { Density (calculated) } & 2 & \\ \text { Absorption coefficient } & 1.516 \mathrm{Mg} / \mathrm{m}^{3} & \\ \text { F(000) } & 0.732 \mathrm{~mm}-1 & \\ \text { Crystal size } & 588 & \\ \text { Theta range for data collection } & 0.45 \times 0.1 \times 0.05 \mathrm{~mm}^{3} \\ \text { Index ranges } & 1.48 \text { to } 25.00^{\circ} . \\ \end{array}$


Reflections collected

Independent reflections

Reflections [I>2sigma(I)]

Completeness to theta $=25.00^{\circ}$

Absorption correction

Refinement method

Data / restraints / parameters

Goodness-of-fit on $\mathrm{F}^{2}$

Final $R$ indices [ $\mathrm{l}>2$ sigma( $(\mathrm{l})$ ]

$R$ indices (all data)

Largest diff. peak and hole
7692

$4394[R$ (int) $=0.0384]$

3531

$99.5 \%$

None

Full-matrix least-squares on $\mathrm{F}^{2}$ 4394 / 0 / 343

1.066

$\mathrm{R} 1=0.0540, w R 2=0.1442$

$\mathrm{R} 1=0.0678, w R 2=0.1539$

0.575 and -0.468 e. $\AA^{-3}$ 


\section{Results and discussion}

\subsection{Synthesis of TTFV}

The TTFV 9 was prepared via the synthetic route outlined in Scheme 2 . In this synthesis, the key intermediate is the aldehyde 6 that was obtained from the known phosphonium salt 5.[27] To a solution of $\mathbf{5}$ and glyoxal $\left(\mathrm{C}_{2} \mathrm{H}_{2} \mathrm{O}_{2}\right)$ in dried acetonitrile, trimethylamine was added to achieve the deprotonation of $\mathbf{5}$ to form the ylide which in situ reacts with glyoxal to form the aldehyde $\mathbf{6}$. Triphenylphosphonium salt $\mathbf{5}$ is not reactive enough to react with the aldehyde $\mathbf{6}$, and for this, the analogous tributylphosphonium salt $\mathbf{8}$ was synthesised. Finally, the desired compound TTFV 9 was obtained through a Wittig reaction between 6 and the phosphorus ylide derived from salt 8 with a $74 \%$ yield.

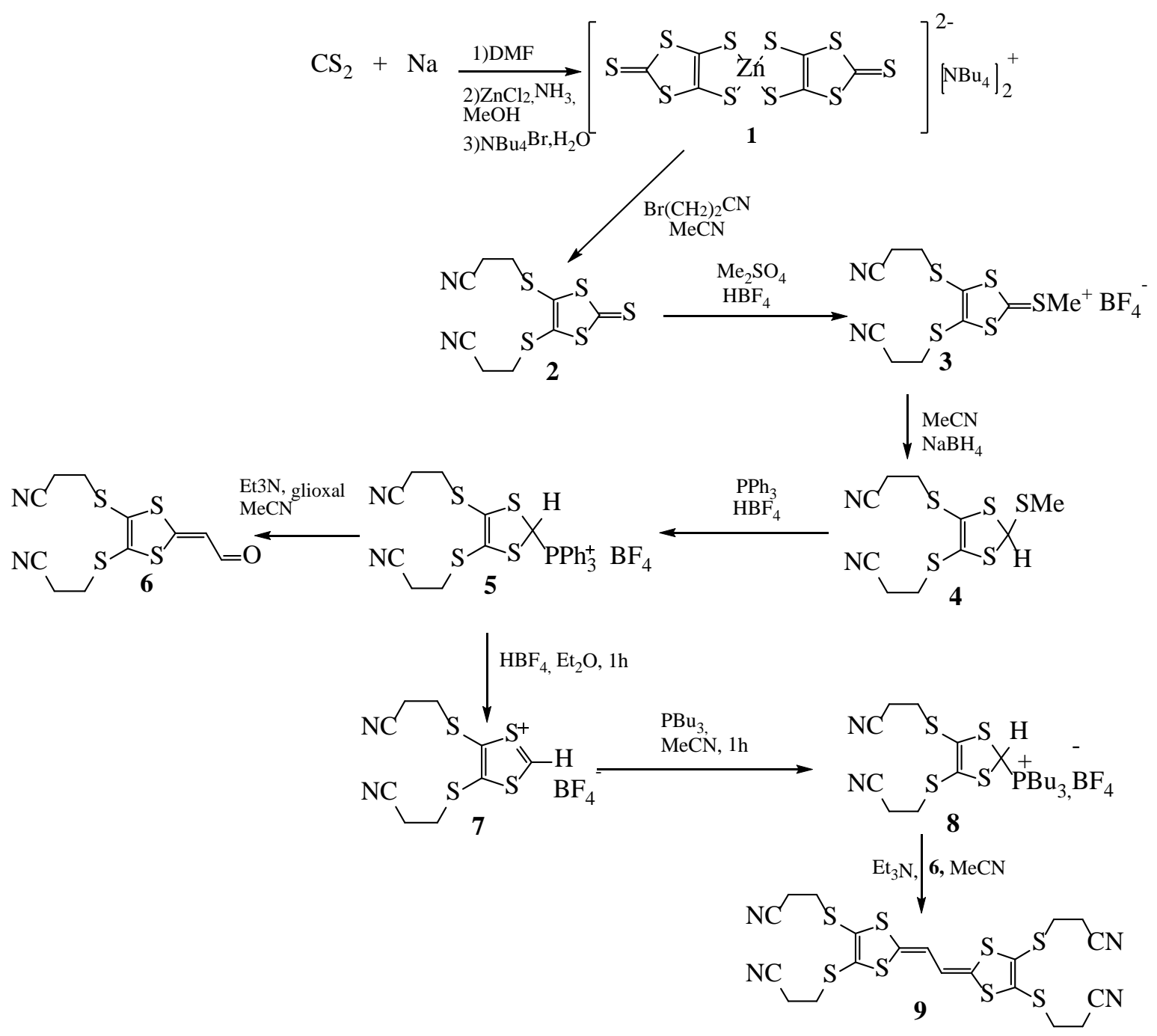




\section{$2.2 X$-ray molecular and crystal structure}

X-ray quality single crystals of $\mathbf{9}$ were grown following two methodologies: i) from slow evaporation in a Petri dish of a $7 \mathrm{mM}$ solution in DMF for several days and, ii) from a slow cooling down of a saturated solution in acetonitrile (ACN). The saturated solution in ACN was heated up to $80^{\circ} \mathrm{C}$ in an oven and then cooled down to room temperature with a rate of $1^{\circ} \mathrm{C}$ per hour. Both crystallization techniques gave rise to pale yellow needle shape crystals with the same crystal structure (Table 1).

Molecules in the crystal of the TTFV 9 show a coplanar $\pi$ system with the four cyanoethyl chains being almost perpendicular to this plane (Figure 1). The structure presents spatial disorder of two $\mathrm{CN}$ groups of the molecule.

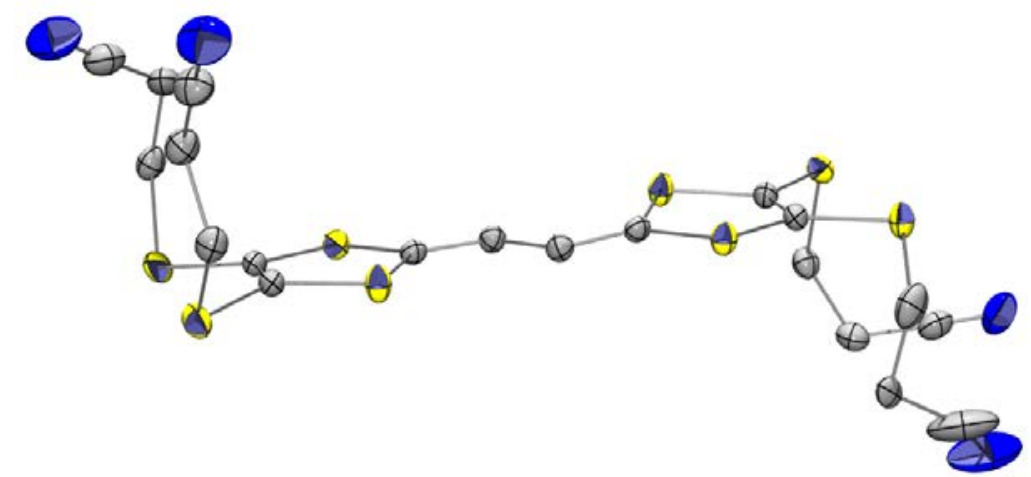

Figure 1 Molecular structure of TTFV 9 in which all hydrogen atoms have been omitted for clarity.

Molecules of $\mathbf{9}$ in the crystal form $\pi$-stacks parallel to the crystallographic $a$ axis with a displacement between them along the longest axis of the molecule that correspond

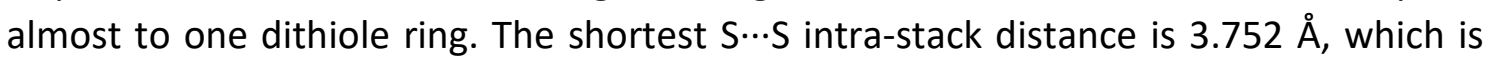
slightly longer that the sum of van der Waals radii $(1.8 \AA)$ and there are no short S...S contacts between the neighbouring stacks.
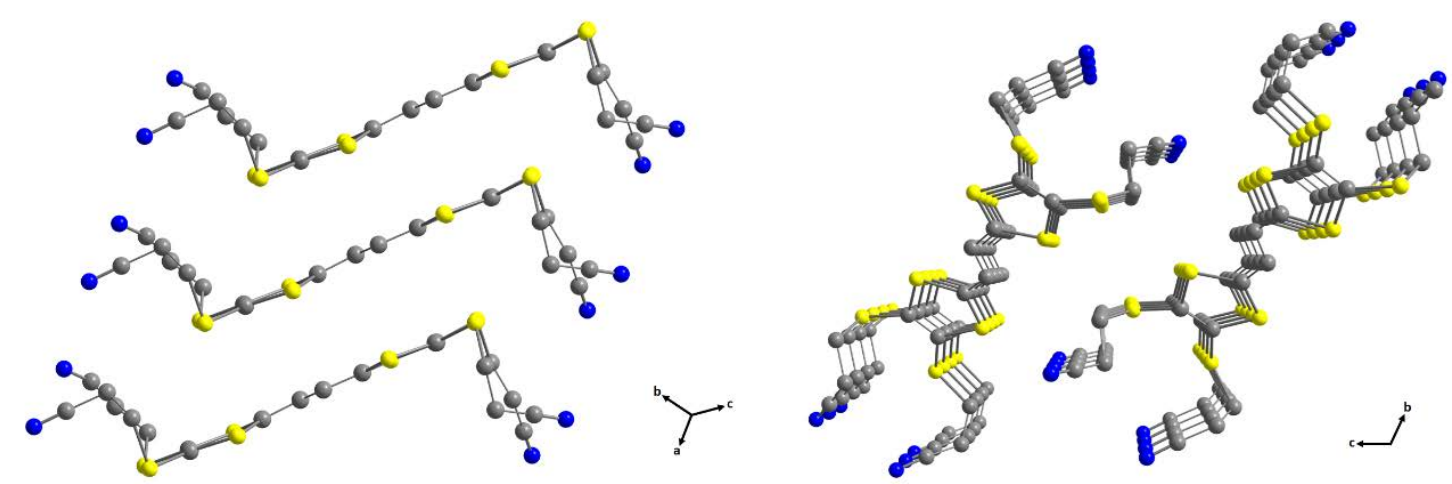

Figure 2 Two views of the crystal structure of 9 showing the $\pi$ - $\pi$ stacking of the central core (left) and the projection along the a axis of the molecule in the unit cell. 


\subsection{Electrochemical characterization and charge transfer compounds preparation.}

The solution redox properties of $\mathbf{9}$ were studied by cyclic voltammetry (CV). The cyclic voltammogram of 9 (Figure 3 ) shows two reversible redox waves at $\mathrm{E}_{1}{ }^{1 / 2}=600 \mathrm{mV}$ and $\mathrm{E}_{2}{ }^{1 / 2}=770 \mathrm{mV}$ (vs. Ag/AgCl; $\mathrm{Fc} / \mathrm{Fc}+\mathrm{E}^{1 / 2}=459 \mathrm{mV}$ ), corresponding to two single-electron redox steps giving to the formation of the cation radical and the dication species, respectively. The CV data show that the TTFV 9 has a good oxidation potential to behave as a stable p-type semiconductor, similar to the well-known and high performing semiconductor dibenzo tetrathiafulvalene.[31,32] Further, there is a small difference $(\Delta E)$ between $E_{1 / 2}^{1}$ and $E^{2} 1 / 2$ which is indicative of an increased charge separation and thus reduced on-site coulombic repulsion.

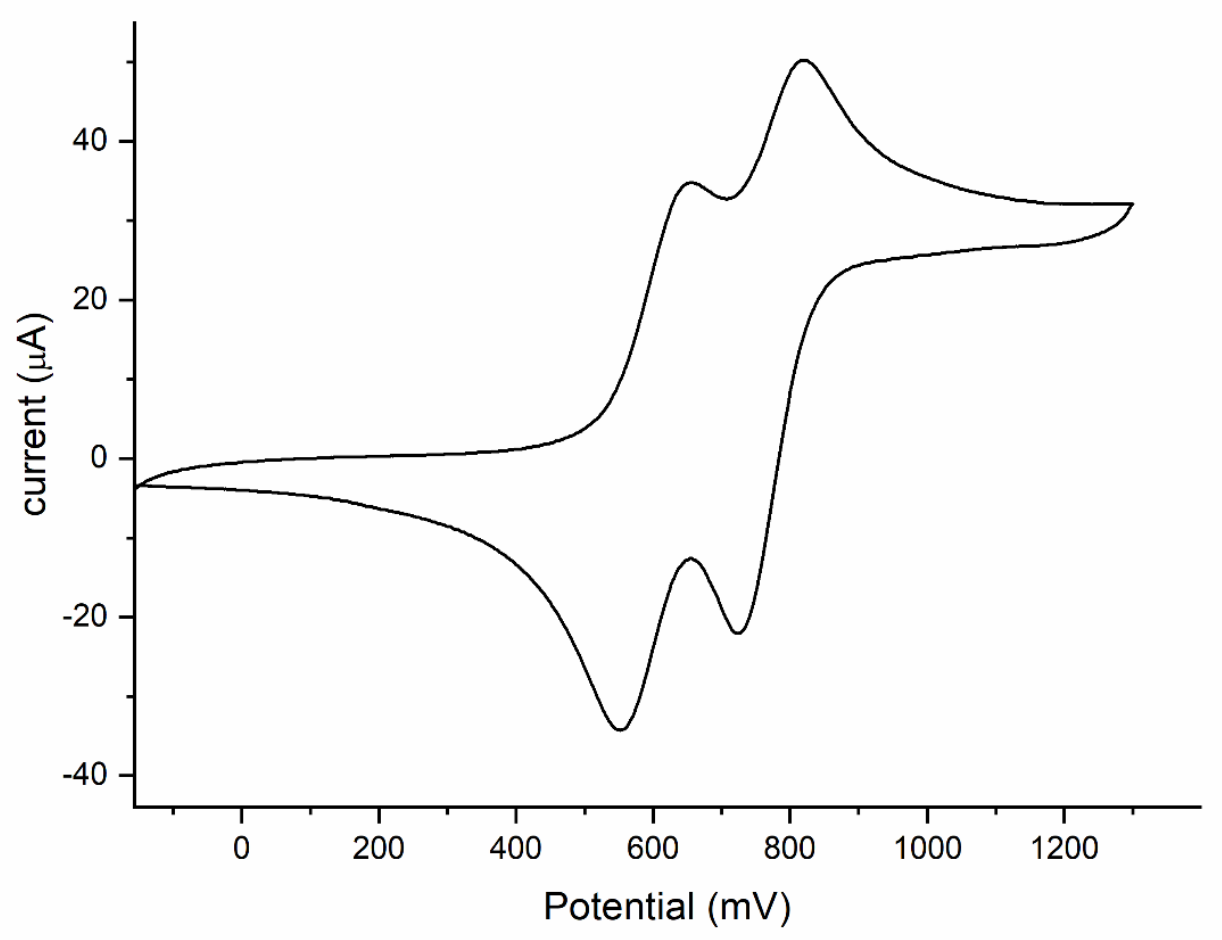

Figure 3 Cyclic voltammetry of 9 in $\mathrm{CH} 2 \mathrm{Cl} 2$ containing 0.1 M Bu4NPF6 at room temperature vs $\mathrm{Ag} / \mathrm{AgCl}$.

Before the preparation of the CT compounds, the properties of the cation radical derived from 9 were studied. Oxidation of the TTFV 9 to the corresponding radical cation was performed in solution of anhydrous ACN by addition of an excess of a solution of $\mathrm{NOBF}_{4}$ in the same solvent under inert atmosphere at room temperature. The solution turned dark and a signal appeared in the Electron Paramagnetic Resonance (EPR) spectrum corresponding to the formation of the cation radical. Figure 4 a shows the EPR of $\mathbf{9}^{+\cdot}$ that consists in three lines centered at $g=2.0073$ arising from the coupling of the free electron with the nuclear spin of the two hydrogens of the central vinylene with a coupling constant $\left(\left|a_{H}\right|=2.74\right.$ gauss), which is very similar to other known vinylogous TTF derivatives.[33] 
When a solution of $9\left(10^{-5} \mathrm{M}\right.$ in $\left.\mathrm{ACN}\right)$ and a solution of the strong acceptor 2,3,5,6tetrafluoro-7,7,8,8-tetracyanoquinodimethane $\left(\mathrm{TCNQF}_{4}\right)$ in the same solvent, were mixed in a equimolar ratio the charge transfer complex was formed as ascertained from the EPR (Figure 4b) and UV-vis spectra. The EPR shows two signals centred at $g=2.0079$ and $g=2.0032$ corresponding to the cation radical $9^{+\cdot}$ and to the anion radical TCNQF $_{4}{ }^{-*}$, respectively.[34] The measured area of both signals is in accordance with the formation of a 1:1 9-TCNQF 4 complex. In the UV-vis absorption spectrum (Figure 5a) it is clearly observed the shift of the $S_{0} \rightarrow S_{1}$ transition of the neutral TCNQ $F_{4}$ at $393 \mathrm{~nm}$ to $405 \mathrm{~nm}$ corresponding to the $D_{0} \rightarrow D_{2}$ transition and the appearing of other local maxima at 749 and $831 \mathrm{~nm}$ due to the $\mathrm{D}_{0} \rightarrow \mathrm{D}_{1}$ transition of the formed specieTCNQF ${ }_{4}^{--} \cdot[14,35]$

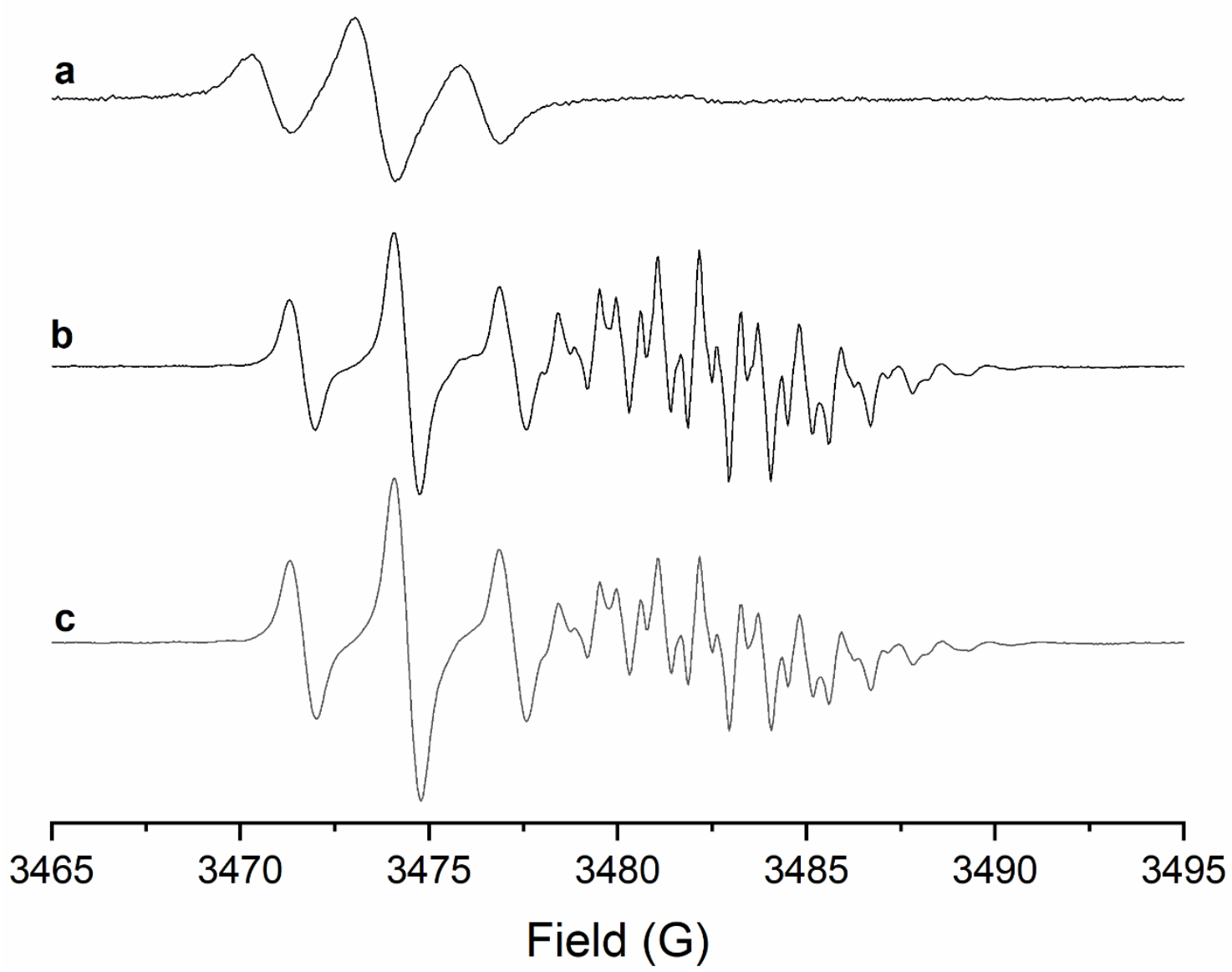

Figure 4 EPR spectrum at room temperature of a) 9+. BF4- in acetonitrile solution b) the CT complex 9 -TCNQF4 formed in acetonitrile solution. c) An acetonitrile solution of the solid CT complex 9 -TCNQF4 formed by physical mixture of both components.

Crystals of the CT complex of 9-TCNQF 4 were obtained by slow evaporation of an equimolar solution of the donor and the acceptor in $\mathrm{CH}_{2} \mathrm{Cl}_{2}$. After overnight evaporation in an open vial, small green crystals were formed (Figure S1 left). Additionally, the crystals were also prepared by the diffusion method. In this case, $3 \mathrm{mg}$ of the TTFV 9 were dissolved in $4 \mathrm{ml}$ of $\mathrm{CH}_{2} \mathrm{Cl}_{2}$ while $3.0 \mathrm{mg}$ of $\mathrm{TCNQF}_{4}$ were dissolved in $2 \mathrm{ml}$ of the same solvent. The latter solution was placed slowly over the solution of the TTFV in a test tube. Immediately the solution mixture changed from yellow to dark green and a 
suspension was formed. After 5 hours the solution turned to lighter green and some precipitate was formed. The test tube was sealed with the glass stopper and after four days the solution was filtered to collect very small amount of the crystals of the CT complex 9-TCNQF 4 (Figure S1 right) that were characterized by IR spectroscopy (Figure S5).

Unfortunately, only very small crystals with not enough quality to resolve the crystal structure were obtained from both crystallization procedures. Further methodologies were employed without any successful results.

Taking into account the difficulty of growing good CT crystals, the study and characterization of the solid CT complex was performed with the sample resulting of the physical mixture of the donor TTFV 9 and the acceptor TCNQF 4 grounded in an agate mortar without the addition of any solvent. $1.9 \mathrm{mg}(3.3 \mathrm{mmol})$ of the donor and $0.85 \mathrm{mg}$ ( $3.1 \mathrm{mmol}$ ) of the acceptor were mixed and grinded in the mortar giving a quick change from the parent colours to dark green (Figure S2), which denotes the CT complex formation. UV-Vis-NIR spectrum of the solid sample (Figure $5 \mathrm{~b}$ ) shows a strong broad band centred at $1380 \mathrm{~nm}$, confirming the formation of the CT complex.

The CT complex was characterized by several spectroscopic techniques. EPR spectrum of the complex 9-TCNQF 4 in acetonitrile solution (Figure 4c) clearly shows the contribution of both components of the complex being very similar to that obtained from the complex formed in solution except for the areas of both signals correlation. The relative concentrations of both components are consistent with the formation of a 3:2 9-TCNQF 4 complex.

Similar experiments were performed with the 2,5-difluoro-7,7,8,8tetracyanoquinodimethane $\left(\mathrm{TCNQF}_{2}\right.$ ) which has lower electron affinity than the TCNQF and in this case, as evidenced by the spectroscopic characterization (Figure S3. EPR), the charge transfer degree was almost negligible (Figure S4, IR). 

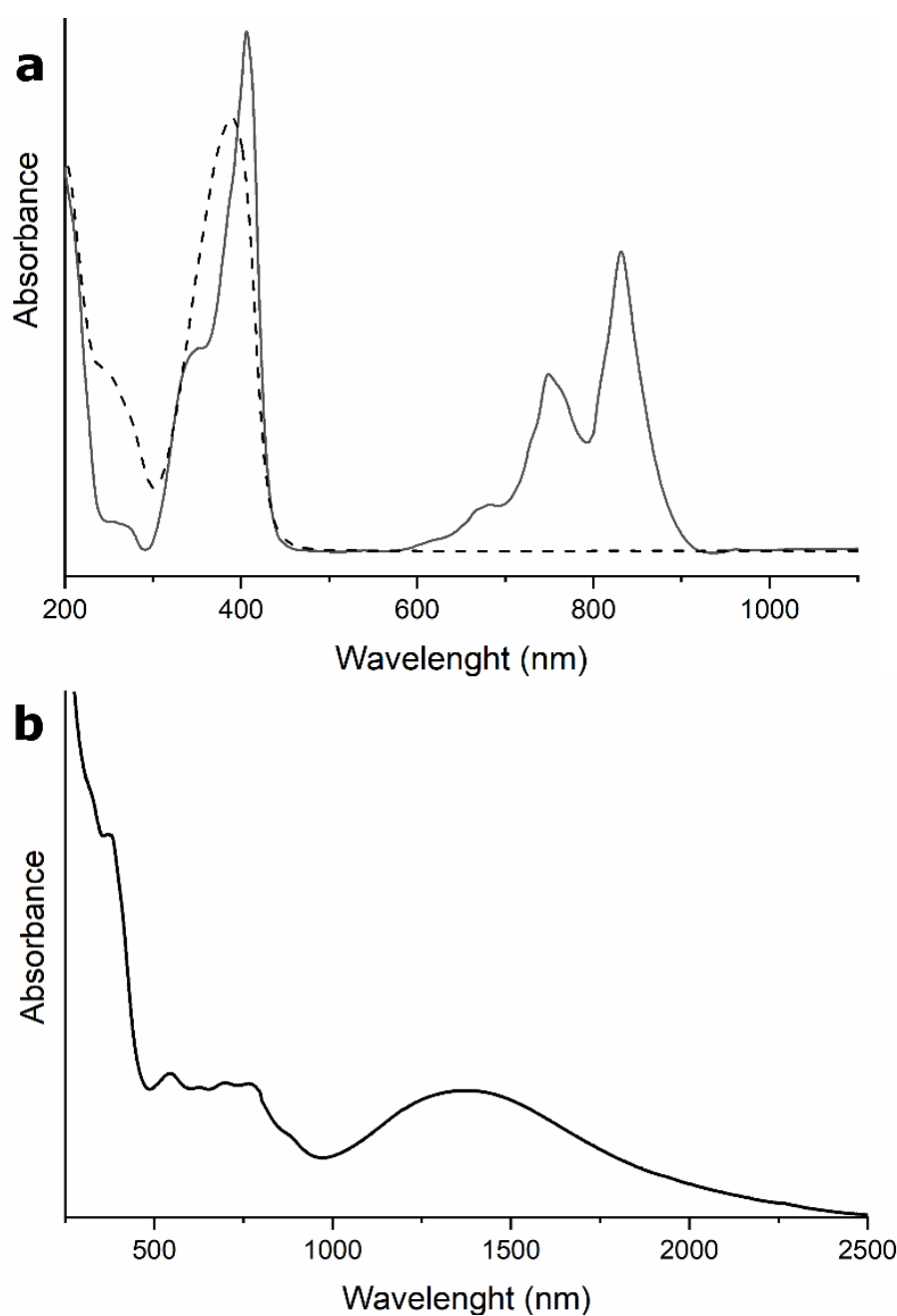

Figure 5 Absorption spectra of a) Acetonitrile solution of $\mathbf{9}$ (dashed line) and CT complex 9-TCNQF 4 (solid line) b) Solid CT complex 9-TCNQF 4 .

\subsection{Estimation of the degree of CT}

The degree of charge transfer $(\rho)$ from the donor to the acceptor moiety in a CT complex is a classical feature to characterize these kind of systems. It has been demonstrated how most of the physical properties of CT complexes are connected with the degree of charge transferred between donor and acceptor molecule but it is still an open issue the standardization of such estimation[6]. Nowadays two methods are commonly used: the bond lenghts distance by X-ray structure and the spectroscopic methods as Raman and Infrared. A comparison between the bond lengths of the neutral species and of the complex shows from X-ray structure that the geometry varies with the electron population of the molecular orbitals. In the specific case of TCNQ and its derivatives, a larger electron population implies a less quinoid character of the molecule, with consequence on the $\mathrm{C}-\mathrm{C}$ bonds distances providing information on $\rho$ through the bond length variation between neutral and charged TCNQs [36,37]. In general this method is the less accurate because of the lack of literature crystallographic data. Further, in our specific case, this methos could not be applied due to the impossibility of obtaining single crystals suitable for the X-ray structural analysis of the salt. 
A more reliable method is represented by the estimation of $\rho$ by looking at the frequency shift of charge sensitive vibrational modes. In our case the system has been identified by EPR measurements as 3:2 ratio between donor and acceptor so we can assume a mixed stack configuration. For mixed stack complexes the Raman active ag vibrations are coupled with the electronic transition and results in an overestimation of the real $\rho .[38]$ The infrared charge sensitive vibrations for mixed stack configuration represent the best choice for the estimation of $\rho$ in donor- acceptor systems.

In previous work by Salzillo et al. $[39,40]$ three charge sensitive vibrations for the TCNQF 4 were identified related to the C-C stretching, which for the pure compound were 1396, 1550 and $1599 \mathrm{~cm}^{-1}$. In Figure 6a the comparison of the IR spectra of 9, TCNQF 4 and the CT complex are reported and one out of three charge sensitive vibrations for the TCNQF 4 in the complex is identified (black arrow, Figure $6 a$ ) as the $v_{2}$ stretching of the $C=C$ double bond group and peaked at $1536 \mathrm{~cm}^{-1}$. Assuming a linear dependence of the charge sensitive vibrations shifts with the ionicity of the complex, we can estimate a $\rho$ of 0.31 .
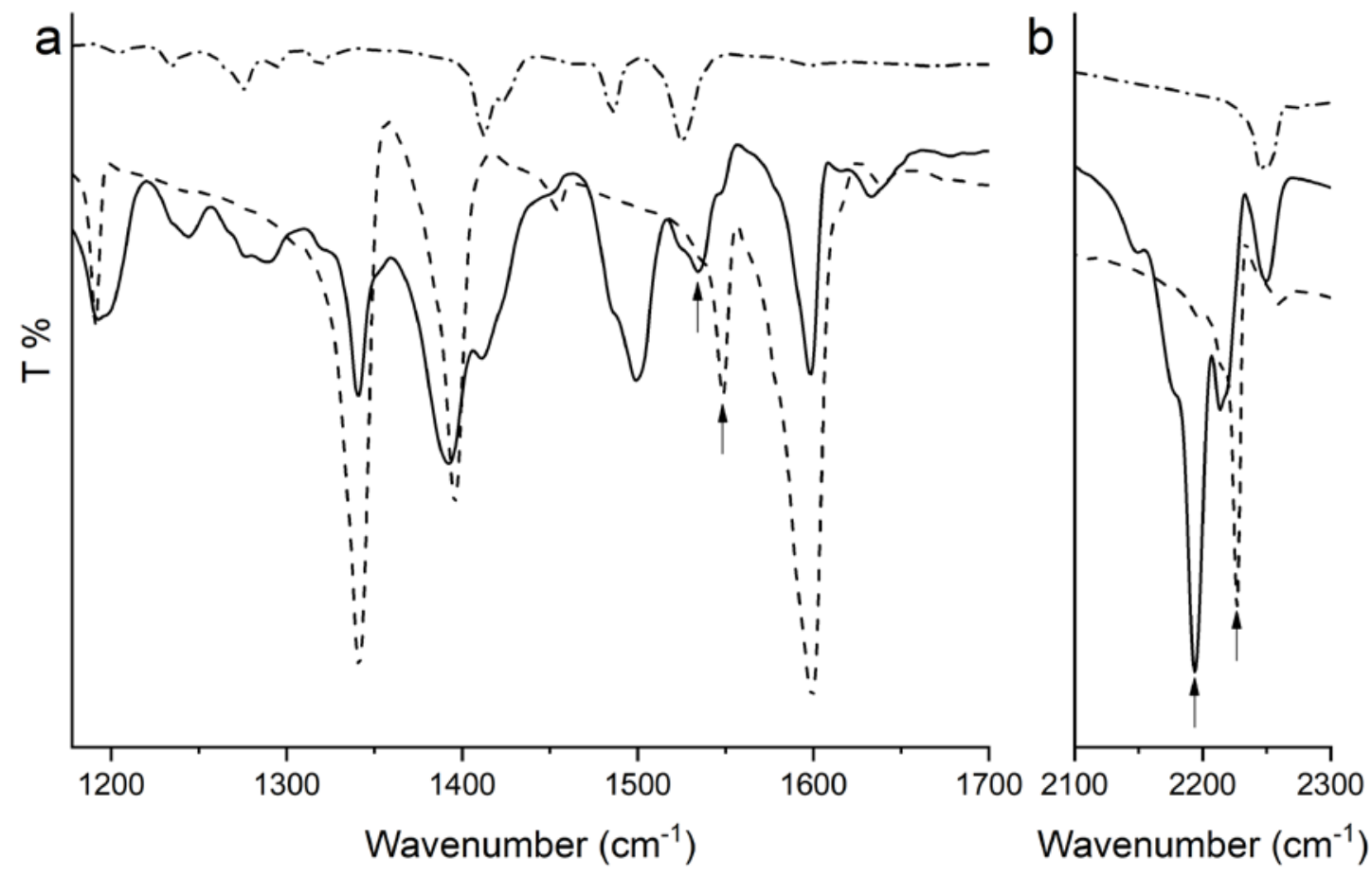

Figure 6 a) Infrared spectra of $\mathbf{9}$ (dash-dot line), $T C N Q F_{4}$ (dash line) and of the charge transfer complex (solid line); $b$ ) $\mathrm{CN}$ group stretching region comparison for the three species.

In figure $6 \mathrm{~b}$ the comparison of the IR spectra in the region of the $\mathrm{CN}$ stretching is illustrated. A strong shift of the CN group of the TCNQF 4 from 2226 to $2194 \mathrm{~cm}^{-1}$ is observed, which confirms the formation of the charge transfer complex. The use of this charge sensitive vibration is often used for the estimation of the ionicity of the CT complex[41] but usually is giving an overestimation due to the external position of such 
functional group and the consequent interaction with the neighbour molecule in the crystal structure.

\section{Conclusions}

A vinylogue TTF derivative bearing four cyanoethylsulfanyl substituents (9) has been synthesized and studied. According to the electrochemical properties, the novel compound shows very good donor properties and a reduced on-site coulombic repulsion. The crystal structure exhibited a planar structure of the conjugated moiety with a slipped $\pi-\pi$ stacking organization in the crystal. Charge transfer complex formation between the TTFV 9 and TCNQF 4 has been corroborated by EPR, UV-Vis and IR spectroscopic studies.

Unfortunately, it has not been possible to grow single crystals of the CT complex 9$\mathrm{TCNQF}_{4}$ suitable for $\mathrm{x}$-ray structural analysis. Nevertheless, a fully spectroscopic study of the donor-acceptor CT complex prepared by mechanochemistry has been performed and used to characterize the system. EPR studies allow for the recognition of the 3:2 stoichiometry ratio between donor and acceptor while IR spectroscopy gave the estimation of the ionicity of the complex, which was found to be 0.31 .

\section{Aknowledgments}

T.S. acknowledges the H2020-MSCA-COFUND-2014 Programme (P-SPHERE, Grant agreement 665919). This work was funded by European research council (ERC) StG 2012-306826 e-GAMES, Networking Research Center on Bioengineering, Biomaterials, and Nanomedicine (CIBER-BBN), the Spanish Ministry project FANCY CTQ2016-80030-R, the Generalitat de Catalunya (2017SGR13) and the Spanish Ministry of Economy and Competitiveness, through the "Severo Ochoa" Programme for Centers of Excellence in R\&D (SEV-2015-0496).

\section{References}

[1] H. Akamatu, H. Inokuchi, Y. Matsunaga, Electrical conductivity of the PeryleneBromine complex, Nature. 173 (1954) 168-169.

[2] J. Ferraris, D.O. Cowan, V. Walatka, J.H. Perlstein, Electron Transfer in a New Highly Conducting Donor-Acceptor Complex, J. Am. Chem. Soc. 95 (1973) 948949. doi:10.1021/ja00784a066.

[3] D. Jérome, Organic conductors: From charge density wave TTF-TCNQ to superconducting (TMTSF)2PF6, Chem. Rev. 104 (2004) 5565-5591. doi:10.1021/cr030652g.

[4] Y. Takahashi, T. Hasegawa, Y. Abe, Y. Tokura, G. Saito, Organic metal electrodes for controlled - and -type carrier injections in organic field- effect transistors 
Organic metal electrodes for controlled $\mathrm{p}$ - and $\mathrm{n}$-type carrier injections in organic field-effect transistors, Appl. Phys. Lett. 88 (2006) 073504.

doi:10.1063/1.2173226.

[5] B.E. Laukhina, R. Pfattner, L.R. Ferreras, S. Galli, M. Mas-torrent, N. Masciocchi, V. Laukhin, J. Veciana, Ultrasensitive Piezoresistive All-Organic Flexible Thin Films, Adv. Mater. 22 (2010) 977-981. doi:10.1002/adma.200902639.

[6] K.P. Goetz, D. Vermeulen, M.E. Payne, C. Kloc, L.E. Mcneil, O.D. Jurchescu, Charge-transfer complexes : new perspectives on an old class of compounds, J. Mater. Chem. C. 2 (2014) 3065-3076. doi:10.1039/c3tc32062f.

[7] R. Pfattner, M. Mas-torrent, field-effect transistor performance, Phys. Chem. Chem. Phys. 17 (2015) 26545-26552. doi:10.1039/C4CP03492A.

[8] E. Collet, M.H. Lemée-Cailleau, M. Buron-Le Cointe, H. Cailleau, M. Wulff, T. Luty, S. Koshihara, M. Meyer, L. Toupet, P. Rabiller, S. Techert, Laser-Induced Ferroelectric Structural Order in an Organic Charge-Transfer Crystal, Science 300 (2003) 612-615. doi:10.1126/science.1082001.

[9] S. Horiuchi, K. Kobayashi, R. Kumai, N. Minami, F. Kagawa, Y. Tokura, Quantum ferroelectricity in charge-transfer complex crystals, Nat. Commun. 6 (2015) 1-7. doi:10.1038/ncomms8469.

[10] M. Mas-Torrent, C. Rovira, Novel small molecules for organic field-effect transistors: Towards processability and high performance, Chem. Soc. Rev. 37 (2008) 827-838. doi:10.1039/b614393h.

[11] R. Pfattner, S.T. Bromley, C. Rovira, M. Mas-torrent, Tuning Crystal Ordering , Electronic Structure, and Morphology in Organic Semiconductors :

Tetrathiafulvalenes as a Model Case, Adv. Funct. Mater. 26 (2016) 2256-2275. doi:10.1002/adfm.201502446.

[12] M.L. Tietze, J. Benduhn, P. Pahner, B. Nell, M. Schwarze, H. Kleemann, M. Krammer, K. Zojer, K. Vandewal, K. Leo, Elementary steps in electrical doping of organic semiconductors, Nat. Commun. 9 (2018) 1182. doi:10.1038/s41467-01803302-z.

[13] B. Lüssem, C.M. Keum, D. Kasemann, B. Naab, Z. Bao, K. Leo, Doped Organic Transistors, Chem. Rev. 116 (2016) 13714-13751. doi:10.1021/acs.chemrev.6b00329.

[14] H. Méndez, G. Heimel, S. Winkler, J. Frisch, A. Opitz, K. Sauer, B. Wegner, M. Oehzelt, C. Röthel, S. Duhm, D. Többens, N. Koch, I. Salzmann, Charge-transfer crystallites as molecular electrical dopants, Nat. Commun. 6 (2015) 8560. doi:10.1038/ncomms9560.

[15] M. Mas-Torrent, C. Rovira, Tetrathiafulvalene derivatives for organic field effect transistors, J. Mater. Chem. 16 (2006) 433-436. doi:10.1039/b510121b.

[16] J. Yamada, T. Sugimodo, TTF Chemistry: Fundamentals and Applications of Tetrathiafulvalenes, Kodansa and Springer, 2014. 
[17] F. Pop, N. Avarvari, Covalent non-fused tetrathiafulvalene-acceptor systems, Chem. Commun. 52 (2016) 7906-7927. doi:10.1039/c6cc01827k.

[18] O. Drozdova, H. Yamochi, K. Yakushi, M. Uruichi, S. Horiuchi, G. Saito, Determination of the charge on BEDO-TTF in its complexes by Raman spectroscopy, J. Am. Chem. Soc. 122 (2000) 4436-4442. doi:10.1021/ja9927523.

[19] C. Rovira, Bis (ethylenethio) tetrathiafulvalene (BET-TTF) and Related Dissymmetrical Electron Donors : From the Molecule to Functional Molecular Materials and Devices (OFETs), Chem. Rev. 104 (2004) 5289-5317.

[20] P. Frère, P.J. Skabara, Salts of extended tetrathiafulvalene analogues: Relationships between molecular structure, electrochemical properties and solid state organisation, Chem. Soc. Rev. 34 (2005) 69-98. doi:10.1039/b316392j.

[21] Y. Kiyota, T. Kadoya, K. Yamamoto, K. Iijima, T. Higashino, T. Kawamoto, K. Takimiya, T. Mori, Benzothienobenzothiophene-Based Molecular Conductors: High Conductivity, Large Thermoelectric Power Factor, and One-Dimensional Instability, J. Am. Chem. Soc. 138 (2016) 3920-3925. doi:10.1021/jacs.6b01213.

[22] Y. Mlsaki, N. Higuchi, H. Fujiwara, T. Yamabe, T. Mori, H. Mori, S. Tanaka, ( DTEDT )[ Au ( CN )2]0.4 : An Organic superconductor based on the novel pielectron Framework of Vinylogous Bis-Fused tetrathiafulvalene, Angew. Chemie - Int. Ed. 34 (1995) 1222-1225.

[23] Z. -i Yoshida, T. Sugimoto, New Donors for Molecular Organic (Super)Conductors and Ferromagnets, Angew. Chemie Int. Ed. English. 27 (1988) 1573-1577. doi:10.1002/anie.198815731.

[24] M.R. Bryce, A. Chesney, S. Yoshida, A.J. Moore, A.S. Batsanov, J.A.K. Howard, Synthesis and $\mathrm{X}$-ray crystal structure of a vinylogue of tetramethyltetraselenafulvalene, J. Mater. Chem. 7 (1997) 381-385.

[25] S. Liang, G. Chen, J. Peddle, Y. Zhao, Reversible dispersion and releasing of single-walled carbon nanotubes by a stimuli-responsive TTFV-phenylacetylene polymer, Chem. Commun. 48 (2012) 3100-3102. doi:10.1039/c2cc17935k.

[26] Y. Zhao, G. Chen, K. Mulla, I. Mahmud, S. Liang, P. Dongare, D.W. Thompson, L.N. Dawe, S. Bouzan, Tetrathiafulvalene vinylogues as versatile building blocks for new organic materials, Pure Appl. Chem. 84 (2012) 1005-1025. doi:10.1351/PAC-CON-11-09-22.

[27] T.K. Hansen, J. Becher, T. Jørgensen, K.S. Varma, R. Khedekar, M.P. Cava, 4, 5Dibenzoyl-1, 3-Dithiole-2-Thione: Benzenecarbothioic acid, S, S'-(2-thioxo-1, 3dithiole-4, 5-diyl) ester, Org. Synth. 73 (1996) 270.

[28] K.B. SImonsen, N. Svenstrup, J. Lau, O. SImonsen, P. Mork, G.J. Kristensen, J. Becher, Sequential Functionalisation of bis-protected tetrathiafulvalenedithiolates, Synthesis (Stuttg). (1996) 407.

[29] L. Binet, J.M. Fabre, C. Montginoul, K.B. Simonsen, J. Becher, L. De Chimie, O. Structurale, U.M. Ii, P.E. Bataillon, Preparation and chemistry of new unsymmetrically substituted tetrachalcogenofulvalenes bearing $\mathrm{CN}(\mathrm{CH} 2), \mathrm{X}$ and 
$\mathrm{HO}(\mathrm{CH}),, \mathrm{X}$ groups $(\mathrm{X}=\mathrm{S}$ or Se), J. Chem. Soc. Perkin Transation. 1 (1996) 783788.

[30] G.M. Sheldrick, SHELXS97 and SHELXL97. Program for Crystal Structure Solution and Refinement., (1997).

[31] M. Mas-Torrent, P. Hadley, S.T. Bromley, N. Crivillers, J. Veciana, C. Rovira, Single-crystal organic field-effect transistors based on dibenzo-

tetrathiafulvalene, Appl. Phys. Lett. 86 (2005) 012110. doi:10.1063/1.1848179.

[32] F.G. Pozo, S. Fabiano, R. Pfattner, S. Georgakopoulos, C. Rovira, X. Crispin, M. Berggren, M. Mas-torrent, Single Crystal-Like Performance in Solution-Coated Thin-Film Organic Field-Effect Transistors, Adv. Funct. Mater. 26 (2016) 23792386. doi:10.1002/adfm.201502274.

[33] M.R. Bryce, A.J. Moore, B.K. Tanner, R. Whitehead, W. Clegg, F. Gerson, A. Lamprecht, S. Pfenninger, Vinylogous tetrathiafulvalene (TTF) pi-electron donors and derived radical cations: ESR spectroscopic, magnetic, and X-ray structural studies, Chem. Mater. 8 (1996) 1182-1188.

[34] F. Gerson, R. Heckendorn, D.O. Cowan, A.M. Kini, M. Maxfield, Radical Anions and Radical Trianions of Tetracyanoarenoquinodimethanes. An ESR and ENDOR Study, J. Am. Chem. Soc. 105 (1983) 7017-7023. doi:10.1021/ja00362a002.

[35] L. Ma, P. Hu, H. Jiang, C. Kloc, H. Sun, C. Soci, A.A. Voityuk, M.E. Michel-beyerle, G.G. Gurzadyan, Single photon triggered dianion formation in TCNQ and F 4 TCNQ crystals, Sci. Rep. 6 (2016) 28510. doi:10.1038/srep28510.

[36] F.M. Wiygul, J.P. Ferraris, J. Emge, T.J. Kistenmacher, Molecular Crystals and Liquid Crystals Crystal, Molecular and Electronic Structure of the Electron Acceptor 2 , 5- TCNQF2, Mol. Cryst. Liq. Cryst. 78 (1981) 279-293. doi:10.1080/00268948108082165.

[37] T.J. Emge, F.M. Wiygul, J.P. Ferraris, T.J. Kistenmacher, Crystal Structure and Madelung Energy for the Charge-Transfer Complex Tetrathiafulvalene-2,5Difluro-7,7,8,8-Tetracyano-P-Quinodimethane, Ttf-2,5-Tcnqf2 - Observations on a Dimerized Segregated Stack Motif., Mol. Cryst. Liq. Cryst. 78 (1981) 295-310. doi:10.1080/00268948108082166.

[38] A. Girlando, R. Bozio, C. Pecile, J.B. Torrance, Discovery of vibronic effects in the Raman spectra of mixed-stack charge-transfer crystals, 26 (1982) 2306-2309.

[39] T. Salzillo, M. Masino, G. Kociok-Köhn, D. Di Nuzzo, E. Venuti, R.G. Della Valle, D. Vanossi, C. Fontanesi, A. Girlando, A. Brillante, E. Da Como, Structure, Stoichiometry, and Charge Transfer in Cocrystals of Perylene with TCNQ-Fx, Cryst. Growth Des. 16 (2016) 3028-3036. doi:10.1021/acs.cgd.5b01663.

[40] J. Henderson, M. Masino, L.E. Hatcher, G. Kociok-Köhn, T. Salzillo, A. Brillante, P.R. Raithby, A. Girlando, E. Da Como, New Polymorphs of Perylene:Tetracyanoquinodimethane Charge Transfer Cocrystals, Cryst. Growth Des. 18 (2018) 2003-2009. doi:10.1021/acs.cgd.7b01391.

[41] J.S. Chappell, W.A. Bryden, M. Maxfield, D.O. Cowan, A.N. Bloch, T.O. Poehler, 
Degree of Charge Transfer in Organic Conductors by Infrared Absorption Spectroscopy, J. Am. Chem. Soc. 103 (1981) 2442-2443.

doi:10.1021/ja00399a066. 